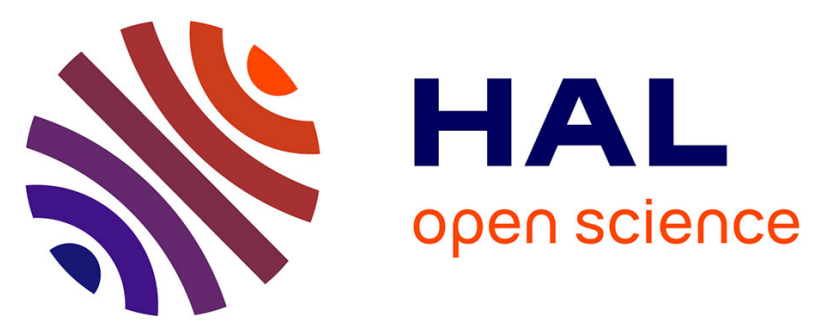

\title{
Z-Axis Controllable Mille-Feuille electrode electrorotation device utilizing levitation effect
}

Yuki Okamoto, Taku Tsuchiya, Charles Moslonka, Yu-Sheng Lin, Sung Tsang, Frédéric Marty, Ayako Mizushima, Chen-Li Sun, Hsiang-Yu Wang, Agnès Tixier-Mita, et al.

\section{To cite this version:}

Yuki Okamoto, Taku Tsuchiya, Charles Moslonka, Yu-Sheng Lin, Sung Tsang, et al.. Z-Axis Controllable Mille-Feuille electrode electrorotation device utilizing levitation effect. Transducers 2019 EUROSENSORS XXXIII, Jun 2019, Berlin, Germany. hal-02151887

\section{HAL Id: hal-02151887 \\ https://hal.science/hal-02151887}

Submitted on 10 Jun 2019

HAL is a multi-disciplinary open access archive for the deposit and dissemination of scientific research documents, whether they are published or not. The documents may come from teaching and research institutions in France or abroad, or from public or private research centers.
L'archive ouverte pluridisciplinaire HAL, est destinée au dépôt et à la diffusion de documents scientifiques de niveau recherche, publiés ou non, émanant des établissements d'enseignement et de recherche français ou étrangers, des laboratoires publics ou privés. 


\title{
Z-AXIS CONTROLLABLE MILLE-FEUILLE ELECTRODE ELECTROROTATION DEVICE UTILIZING LEVITATION EFFECT
}

\author{
Yuki Okamoto ${ }^{1}$, Taku Tsuchiya ${ }^{1}$, Charles Moslonka ${ }^{2}$, Yu-Sheng Lin $^{3}$, Sung Tsang ${ }^{4}$,Frédéric Marty, \\ Ayako Mizushima ${ }^{1}$, Chen-li Sun ${ }^{4}$, Hsiang-Yu Wang ${ }^{3}$, Agnès Tixier-Mita ${ }^{1}$, Olivier Français ${ }^{4}$, \\ Bruno Le Pioufle ${ }^{2}$, and Yoshio Mita ${ }^{1}$ \\ ${ }^{1}$ The University of Tokyo, Tokyo, Japan \\ ${ }^{2}$ ENS Paris-Saclay, Cachan, France \\ ${ }^{3}$ National Tin-Hua University, Hsinchu, Taiwan \\ ${ }^{4}$ National Taiwan University, Taipei, Taiwan \\ ${ }^{5}$ ESIEE Paris Université Paris-Est, Noisy le grand France
}

\begin{abstract}
This paper presents a new electrorotation (ROT) device composed of thick walls and multi-electrode layers, called as mille-feuille electrode device. The proposed device has three advantages: (1) The height of a measured particle is controllable by adjusting levitation forces. (2) The rotation of the particle is not affected by the friction to the substrates. (3) The number of electrode layers is extensible.
\end{abstract}

\section{KEYWORDS}

Electrorotation, dielectrophoresis, single cell analysis, microfluidics

\section{INTRODUCTION}

Non-invasive measurement and analysis of a living single cell is necessary in many biological applications. For example, in the case of biofuel production from microalgae cells in culture, the rapid and non-invasive single-cell analysis is required to quantify the amount of intracellular lipids prior to the extraction process [1]. Such characterization and analysis of the cell content can be achieved in a non-invasive way through its dielectric properties. In the case of microalgae, for example, those properties are directly dependent on the lipid content within cells. Impedance spectroscopy and electrorotation (ROT) are commonly used techniques for the measurement of the dielectric properties. Impedance spectroscopy measures the electrical impedance of an analyte, versus the electrical frequency of the applied electric field. However, when measuring the impedance of a micro-object within a microfluidic environment, the accuracy of impedance spectroscopy depends on the dimension of microchannels and electrodes [2,3]. On the other hand, ROT technique is independent on the volume factor (volume of cell versus the volume of the analyte) [3] and renders possible the dielectric properties characterization without a high degree of confinement of the cell between the electrodes. Conventional ROT measurement utilizes four-electrodes, where the measured particle is trapped at the center of the electrode set by negative dielectrophoresis (DEP) forces and then rotated by phase shifted four signals [4]. In ROT measurement, the dielectric properties are extracted by comparing the measured ROT spectra (cell mechanical velocity versus the electrical frequency) and the theoretical model, using least-square minimization algorithms.

Electrodes of a typical ROT device are patterned in a thin metal layer shown in Fig. 1a [5]. In this structure, there are two bottlenecks that degrade the precision of the ROT characteristic: one is friction between the particle and the top or bottom substrates, and the other is the levitation forces caused by variation of the electric field with the cell altitude. Friction affects the rotation velocity. Levitation causes variation of the applied electric field modulus (and thus modify the strength of the ROT torque), and also causes the difficult focusing of the cell by the video recording system. Indeed, with the thin-electrode structure, the electric field is only generated on the bottom substrate. Therefore, the real electric field distribution varies with the altitude, which results to a gradually rise of the cell during the measurement. Consequently, the particle might even reach the top glass substrate, which generates large friction However, if the electric field is decreased to moderate the non-uniformity, the cell remains on the bottom substrate which also generates high friction forces. Moderate levitation is therefore desired in order to levitate the cell between bottom and top substrates.

To decrease such drawbacks effects, thick electrode ROT devices shown in Fig. $1 \mathrm{~b}$ are often proposed to obtain uniform electric field [6, 7]. In such architecture of the device, the insulating channel wall and thin electrodes is replaced by thick electrodes which are electroplated. However, with such technology the electric field is not that uniform, due to the tilting of electrodes (Fig 1b) that appears when electroplating those. The tilting of the thick electrodes causes - once again - the cell levitation towards the top glass substrate.

A better solution is to introduce double-sided electrode structure shown in Fig. 1c, which allows the precise control of the altitude of the cell. This structure is composed of an insulating channel wall sandwiched by two levels of thin electrodes. By applying voltages on top and bottom electrode sets, the altitude of the particle is kept at the midway of the channel due to their repulsive force [8]. However, the fabrication of such device requires a complete encapsulation by permanent flip-chip wafer bonding. Besides, a complex electric and fluidic connection are required. Moreover, the cell height is fixed at one level, it cannot utilize the free-friction space at the other altitudes. 


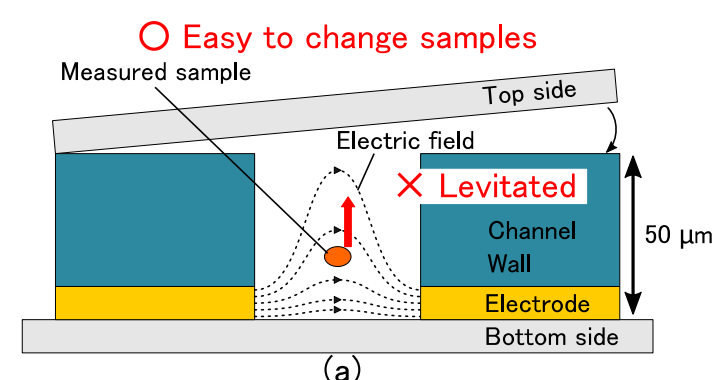

(a)

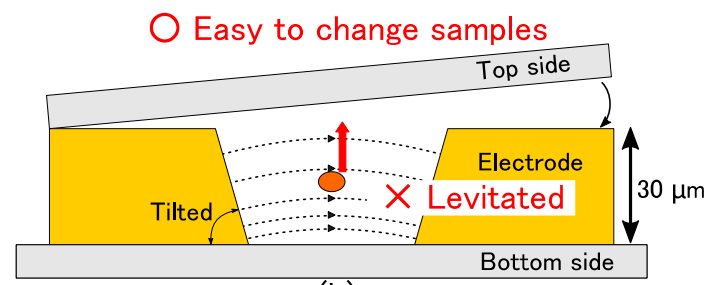

(b)

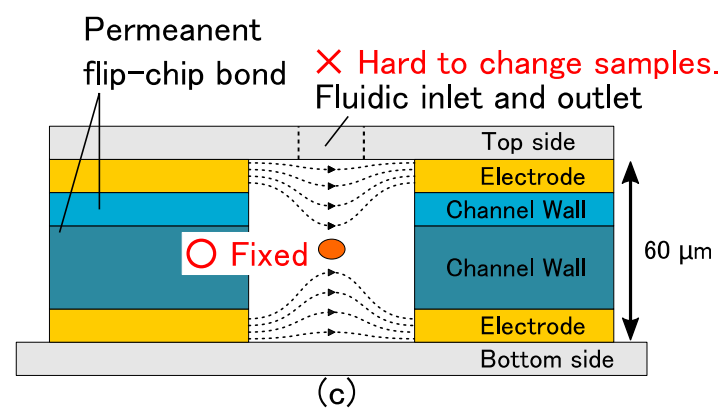

Figure 1: Schematics of (a) conventional thin-electrode, (b) thick-electrode, and (c) double-sided electrode electrorotation device. In the thin and thick electrodes, sample gradually rises because of the asymmetry of electric field. In double-sided electrode device, levitation effect can be controlled, but changing samples is hard.

In this paper, we describe a novel multi-electrodelayer ROT device. Such device allows the levitation of a particle or cell at a precise altitude, defined by the electrical layers that are selected. The device is monolithic and thus does not require complex flip-chip, bonding and complex double side fluidic or electrical connections.

\section{DEVICE DESCRIPTION}

Figure 2a shows the proposed multi-electrode-layer ROT device. The device is monolithic and does not require any complex assembly of processed sub-components. All the layers are successively deposited on the same bottom substrate. The z-directional forces are applied to the cell from each of the upper and lower electrodes. The z-directional (levitation) force generated by each of the electrodes set (in the case of figure $2 b$, the top layer electrodes and the bottom layer electrodes respectively) is expressed as $[8,9]$ :

$$
\left\langle F_{z}\right\rangle=\pi \varepsilon_{m} r^{5} \operatorname{Re}\left[\tilde{f}_{C M}\right] \frac{\partial}{\partial z}\left[\frac{\partial E_{z, \mathrm{rms}}}{\partial z}\right]^{2}
$$

where $\varepsilon_{r}$ is the permittivity of medium, $r$ is the radius of the cell, and $E_{z, \mathrm{rms}}$ is the z-directional root mean square of the electric field. The polarization coefficient $\tilde{f}_{C M}$ is given

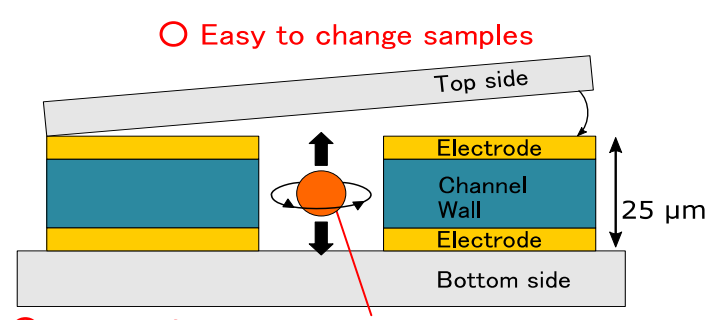

Height of a measured particle can be controlled.

(a)

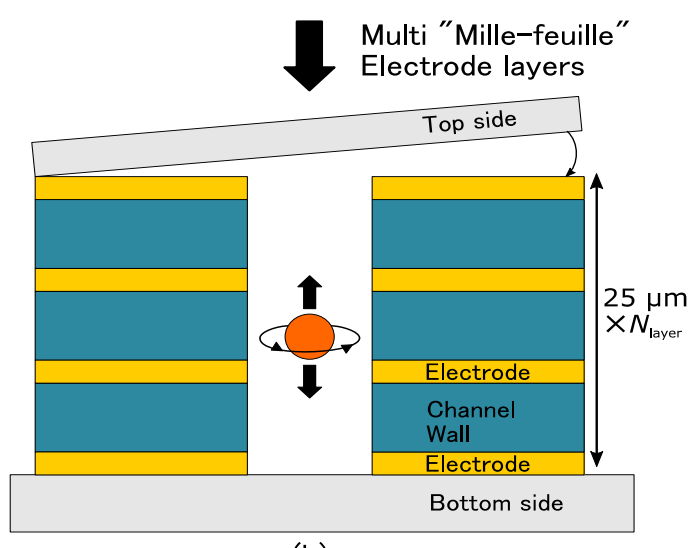

(b)

Figure 2: (a) Schematic of proposed method. This method does not need permanent encapsulation. The height of a particle can be controlled. (b) Schematic of "mille-feuille" structure composed of stacked proposed structures by the same protocol.

Table 1: Comparison of characteristics in previous electrorotation devices and this work.

\begin{tabular}{|c|c|c|c|c|}
\hline & $\begin{array}{c}\text { Thin } \\
\text { Electrode } \\
{[2]}\end{array}$ & $\begin{array}{c}\text { Thick } \\
\text { Electrode } \\
{[4,5]}\end{array}$ & $\begin{array}{c}\text { Double-sided } \\
{[6]}\end{array}$ & This work \\
\hline Friction & Large & Medium & Small & Small \\
\hline $\begin{array}{c}\text { Electric } \\
\text { Field }\end{array}$ & Fixed & Fixed & Fixed & Controllable \\
\hline $\begin{array}{c}\text { Particle } \\
\text { Height }\end{array}$ & Levitated & Levitated & Controllable & Controllable \\
\hline $\begin{array}{c}\text { Sample } \\
\text { introduction }\end{array}$ & Easy & Easy & Difficult & Easy \\
\hline $\begin{array}{c}\text { Channel } \\
\text { Height }\end{array}$ & $\mathbf{5 0 \mu \mathrm { m }}$ & $\mathbf{5 0} \boldsymbol{\mu m}$ & $\mathbf{6 0} \mu \mathrm{m}$ & $\mathbf{2 5} \boldsymbol{\mu \mathrm { m }}$ \\
$\times \boldsymbol{N}_{\text {layer }}$ \\
\hline
\end{tabular}

by following equation:

$$
\tilde{f}_{C M}=\frac{\tilde{\varepsilon}_{c}-\tilde{\varepsilon}_{m}}{2 \tilde{\varepsilon}_{c}+3 \tilde{\varepsilon}_{m}}
$$

where $\tilde{\varepsilon}_{c}$ and $\tilde{\varepsilon}_{m}$ are the complex permittivities of the trapped particle and the medium, respectively. The complex permittivity of a material is given by

$$
\tilde{\varepsilon}=\varepsilon-j \frac{\sigma}{\omega}
$$

where $\varepsilon$ is the permittivity, $\sigma$ is the conductivity, $j$ is the imaginary unit, and $\omega$ is the angular frequency of DEP. As a result, the z-directional force applied to the particle is given by:

$$
F_{z}=F_{z, \text { bottom }}+F_{z, t o p}+F_{g}
$$

where $F_{z, \text { bottom }}$ and $F_{z, \text { top }}$ are the levitating force from the bottom and top electrodes following the equation (1), and 


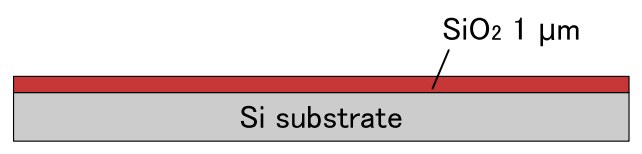

(a)

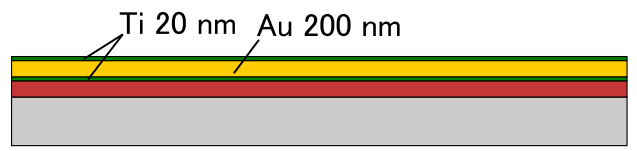

(b)

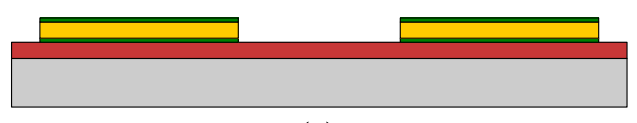

(c)

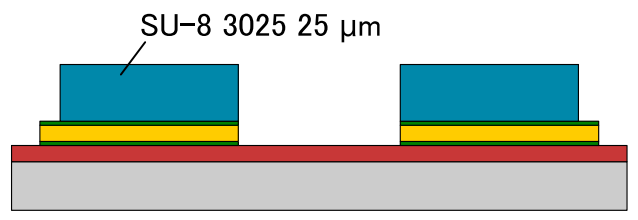

(d)

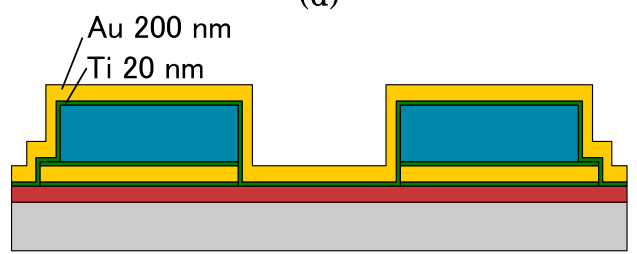

(e)

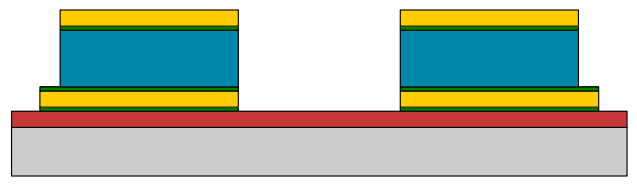

(f)

Figure 3: Process flow of the proposed device. (a) The silicon wafer is oxidized by $1 \mu \mathrm{m}$. (b) The bottom electrode layer of Ti, Au, and Ti are sputtered. (c) Each bottom electrode layer is wet-etched. (d) Channel wall of SU-8 3025 was patterned. (e) The top electrodes of Ti and Au are sputtered. (f) The top electrode layers were patterned.

$F_{g}$ is the gravity of the particle. In the proposed electrode-sandwiching structures shown in Fig. 2a, by adjusting $F_{z, \text { bottom }}$ and $F_{z, \text { top }}$ independently, the altitude of the measured particle is controlled. In other words, it allows us to control friction, electric field, and particle altitude without any limitation. In addition, since the proposed structure does not require any microfluidic operation to introduces the measured particles, changing samples becomes simple. Moreover, the proposed structure has another advantage of extensibility to a "mille-feuille" structure by the same protocol as shown in Fig. 2b. As the mille-feuille structure has multiple electrode layer, it is possible to integrate the sensing electrode layer between the ROT electrodes. Besides, the channel height is independent on material properties and fabrication steps, the mille-feuille method can cope with various sized particles. Table 1 shows the comparison of the characteristics between the previous researches and the proposed structure.

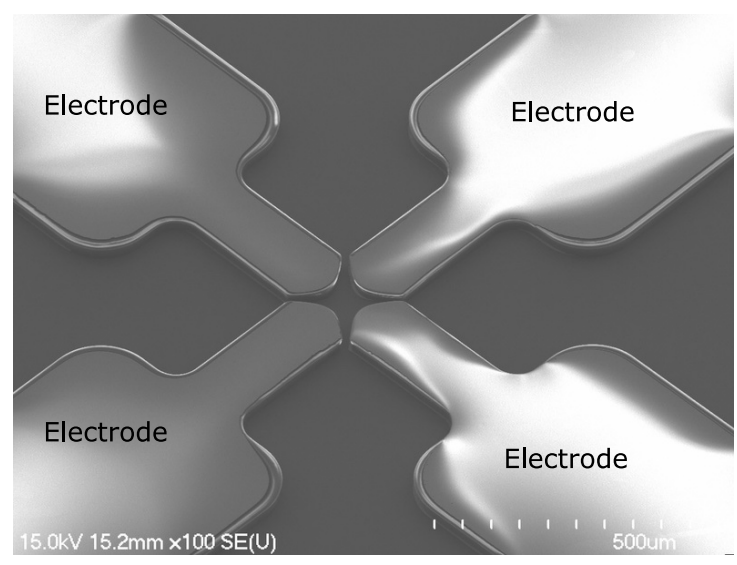

(a)

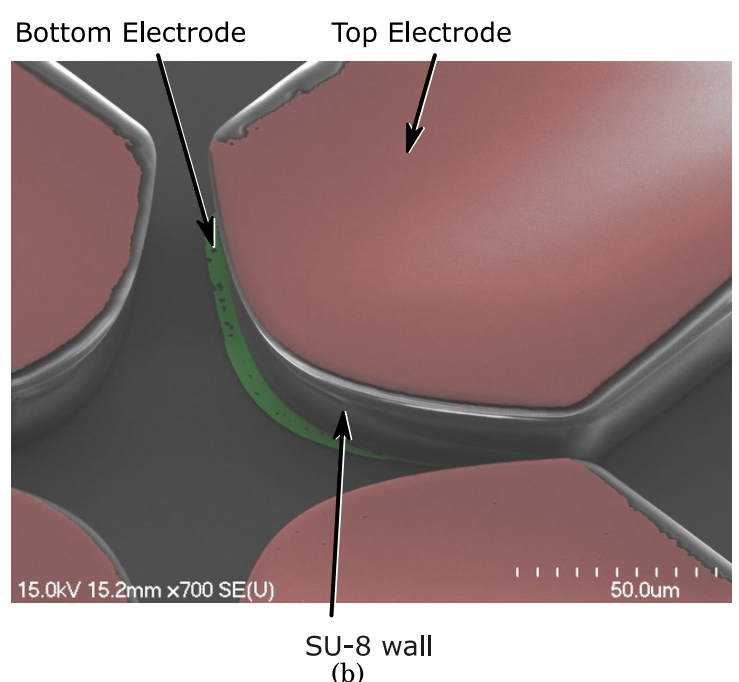

Figure 4: SEM Images of electrorotation device. Figure (a) shows the center of the four electrodes composed of double-side electrodes and thick SU-8 walls. Figure (b) has false color added to highlight the different layers in the structure. Red is top electrode, and green is bottom electrode.

\section{FABRICATION}

Figure 3 shows the processes of the proposed device. We used a $525-\mu \mathrm{m}$-thick silicon wafer. First, the silicon wafer was oxidized by $1 \mu \mathrm{m}$. Next, 20 -nm-thick titanium, 200-nm-thick gold, and 20-nm-thick titanium were sputtered for the bottom electrode. The last titanium layer works as an adhesive layer to SU-8 3025. After that, the negative photoresist Zeon ZPN1150 was patterned, and the titanium and gold layers were etched using 5\% APM and gold etchant (KANTO CHEMICAL AURUM-302), respectively. After the photoresist was removed by acetone, the $25-\mu \mathrm{m}$-thick SU-8 3025 was patterned. Then, for the top electrode, 20-nm-thick titanium and 200-nm-thick gold were sputtered. Subsequently, negative photoresist ZPN1150 was patterned and these electrode layers were etched in the same way as the bottom electrode. Finally, the photoresist was stripped by acetone.

\section{RESULTS}

Figure 4a shows the SEM image of the fabricated proposed device. Figure $4 \mathrm{~b}$ shows the close-up view of the channel wall structure sandwiched by the bottom and top 


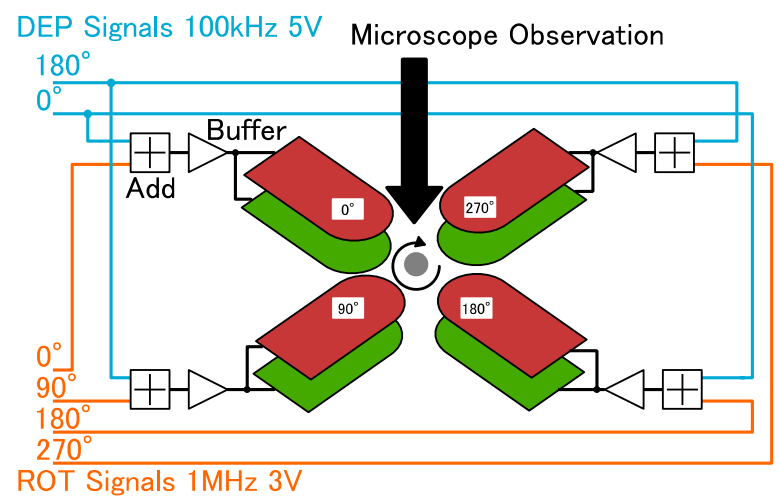

Figure 5: Setup of the experiment. DEP and ROT signals are added by the external circuit. To confirm the levitating force, the same voltages were applied to the top and bottom electrodes. The rotation was observed through a microscope.

\section{$5 \mu \mathrm{m}$ micro bead}

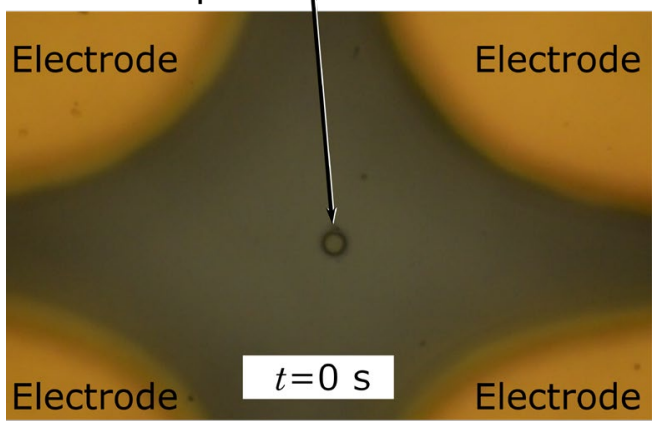

(a)

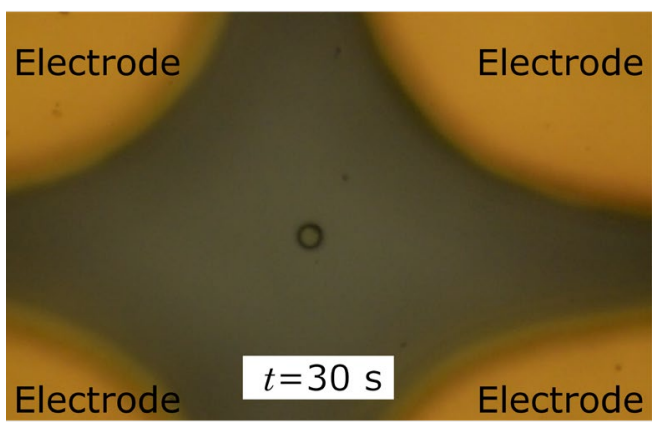

(b)

Figure 6: Images of the micro bead at (a) $t=0 \mathrm{~s}$ and (b) $t=30 \mathrm{~s}$ after rotation started. Thanks to the levitating force from the both sides, the micro bead was trapped at the center of electrodes without changing the levitating height.

electrodes. Figure 5 shows the setup of the experiment. We observed rotation of a $5-\mu \mathrm{m}$ polystyrene micro bead in DI-water through microscope. The bead is introduced using a micropipette and then $100-\mu \mathrm{m}$-thick glass cover is put on the device as the top substrate. DEP signals and ROT phase shifted signals are applied after mixed by the external circuit. We used $100 \mathrm{kHz} 5 \mathrm{~V}$ AC signal for DEP and $1 \mathrm{MHz} 3 \mathrm{~V}$ AC signal for ROT. When the voltages were applied to the bottom electrode, the rise of the measured bead is observed, whereas when we applied the voltages on the top electrodes, the bead fell down. Then, we applied the voltages to both the top and bottom electrodes to confirm if the bead is trapped at the center of the electrodes and rotate it stably. Figure 6 shows the images of the micro bead at $t=0 \mathrm{~s}$ and $t=30 \mathrm{~s}$ after the ROT started. Owing to the levitating force from the both sides, the micro bead was trapped at the center of electrodes without changing the levitating height.

\section{CONCLUSIONS}

We have proposed a novel multi-electrode-layer structure for ROT experiment, which avoids any friction of the characterized micro-object with the top of bottom substrate, due to the precise control of the object levitation. For the first time, the microfabrication of such a device is proposed without any assembly step. The device is monolithic, all layers being successively grown and patterned on the same substrate. . This method has extensibility to stack the wall and electrode layers the proposed structure to mille-feuille structure that allows us to integrate the sensing electrode layer between the ROT electrode layers. Using the fabricated device, we confirmed that a $5-\mu \mathrm{m}$ polystyrene bead was successfully trapped and rotated at the center by applying the DEP and ROT voltages to the bottom and top electrodes without out-of-focus phenomena.

\section{ACKNOWLEDGEMENTS}

The CAD patterns were designed with Cadence Virtuoso, accessible through the academic program of VLSI Design and Education Center (VDEC), The University of Tokyo. The patterning of the device was carried out using the open facilities maintained by Nanotechnology Platform Program of MEXT, and the photomask was fabricated using the VDEC 8-inch EB writer F5112+VD01 donated by Advantest Corporation. This project is jointly supported by JSPS KAKENHI (No. 16H04345 and No. 17J04382) and JSPS-CNRS bilateral joint research project.

\section{REFERENCES}

[1] P. Spolaore, et al., J. Biosci. Bioeng., Vol. 101, Issue 2, pp. 87-96, 2006.

[2] J. Chen, et al., Biomicrofluid., Vol. 5, No. 1, pp. 014113, 2011.

[3] O. Français and B. Le Pioufle, Single Cell Electrical Characterization Techniques, In D. Miklavčič (eds) Handbook of Electroporation, Springer, Cham, pp. 271-288, 2017.

[4] K. Khoshmanesh, et al., Biosens. Bioelectron., vol. 26, no. 5, pp. 1800-1814, 2011.

[5] C. I. Trainito, et al., Electrophoresis, 36, Issues. 9-10, pp. 1115-1122, 2015.

[6] G. Bahrieh, et al., Electrophoresis, vol. 36, pp. 1785-1794, 2015.

[7] M. Eguchi, et al., WAC, 2018, pp. 136-139.

[8] S.-I. Han, et al., Analyst, vol. 138, no. 5, p. 1529, 2013.

[9] T.B. Jones and M. Washizu, J. Electrostat., Vol. 37, Issues 1-2, pp. 121-134, 1996,

\section{CONTACT}

*Y. Okamoto, tel: +81-3-5841-6730;

mems@if.t.u-tokyo.ac.jp 